\title{
Sexo sem tabu: relato de um caso de ação comunitária, com foco na promoção de saúde de adolescentes, em uma escola técnica na cidade de São Paulo
}

\author{
Sex without taboo: report of a community action focused on health promotion \\ for teenagers in a technical high school in the city of São Paulo
}

\section{Bruna Fernandes de Barros ${ }^{1}$, Eduardo Farias Aurélio ${ }^{1}$, Daniela Andrea Medina Macaya ${ }^{1}$, Agatha Mol Marcelo', Deborah Yukiko Otto ${ }^{1}$, Itamar de Souza Santos ${ }^{2}$}

\begin{abstract}
Barros BF, Aurélio EF, Macaya DAM, Marcelo AM, Otto DY, Santos IS. Sexo sem tabu: relato de um caso de ação comunitária, com foco na promoção de saúde de adolescentes, em uma escola técnica na cidade de São Paulo / Sex without taboo: report of a community action focused on health promotion for teenagers in a technical high school in the city of São Paulo. . Rev Med (São Paulo). 2013 abr.jun.;92(2):128-33.
\end{abstract}

\begin{abstract}
RESUMO: A sexualidade, apesar de sua relevância no âmbito da promoção da saúde, é pouco abordada durante os cursos de graduação e por profissionais da saúde na prática clínica. O objetivo deste trabalho é descrever o projeto "Sexo sem Tabu", realizado por alunos do primeiro ano do curso de Medicina da Universidade de São Paulo, dentro da proposta da disciplina "Atenção Primária à Saúde I". Após delineamento do tema e revisão literária, foram executados dois dias de intervenção. Baseados em dinâmicas propostas pelo Ministério da Saúde, discutimos sexualidade e temas correlatos em saúde, com pequenos grupos de alunos de uma escola técnica na cidade de São Paulo. Dentre os alunos participantes, 115/117 (98,3\%) disseram ter dúvidas solucionadas durante a discussão e 114/117 $(97,4 \%)$ consideraram que as informações que foram trazidas eram relevantes para suas vidas. Os alunos da Faculdade de Medicina relataram a oportunidade de treinar várias habilidades afetivas e cognitivas como habilidades de comunicação, desenvoltura, capacidade de lidar com o imprevisto, além de reconhecer que a abordagem da sexualidade não se restringe aos aspectos relacionados às doenças sexualmente transmissíveis, mas também a questões emocionais, morais e fisiológicas. Em conclusão, esta ação comunitária, com foco em promoção da saúde dos adolescentes e abordando o tema da sexualidade, trouxe resultados positivos para os alunos do ensino médio e para a formação profissional dos estudantes de medicina.
\end{abstract}

DESCRITORES: Pormoção da saúde; Sexualidade; Adolescente; Educação médica; Participação comunitária;.

\begin{abstract}
Although sexuality has an evident importance in health promotion, it is rarely discussed during undergraduate courses or assessed by health professionals during clinical practice. The aim of this article is to describe the "Sexo sem Tabu" project, a community action realized by first year medical students from the Faculdade de Medicina da Universidade de São Paulo, during the course "Primary Care I" ("Atenção Primária à Saúde I"). After establishing project design and adequate literature review, we realized two days of community action. Based on dynamics proposed by the Brazilian Ministry of Health, we discussed sexuality and other related health topics within small groups of students from a technical high school in the city of São Paulo. Among high school participants, 115/117 $(98.3 \%)$ considered that they had their doubts solved during the discussion and 114/117(97.4\%) found the information was relevant for their lives. First year medical students documented the opportunity to train several cognitive and affective abilities such as communication, resourcefulness, and how to interact in uncontrolled situations. Furthermore, they recognized that sexuality was not restricted to sexually transmitted diseases, but included emotional, ethical and physiological issues. Concluding, this community action, focused on health promotion for teenagers assessing themes on sexuality, brought positive results for high school students and for the training of medical students.
\end{abstract}

KEYWORDS: Health promotion; Sexuality; Adolescent; Students, medical; Consumer participation

\footnotetext{
${ }^{1}$ Alunos do $2^{\circ}$ ano da graduação em Medicina da Faculdade de Medicina da Universidade de São Paulo.

${ }^{2}$ Professor Doutor da Disciplina de Clínica Geral e Propedêutica do Departamento de Clínica Médica da Faculdade de Medicina da Universidade de São Paulo.

Endereço para correspondência: Itamar S. Santos. Center for Clinical and Epidemiological Research. Hospital Universitário da Universidade de São Paulo. Av. Professor Lineu Prestes, 2565 - $3^{\circ}$ andar. Cidade Universitária. São Paulo, SP, Brazil. 05508-000. e-mail: itamarss@usp.br
} 


\section{INTRODUÇ̃̃O}

sexualidade é um tema importante a
ser abordado na promoção à saúde,
especialmente com adolescentes e jovens ${ }^{1}$. Todavia, esse assunto é tradicionalmente pouco discutido durante a graduação. As diretrizes curriculares nacionais para o curso médico não trazem menção direta à temática sexual ${ }^{2}$.

Pesquisa realizada na Faculdade de Medicina de Botucatu revelou que, embora os alunos do sexto ano de medicina possuam amplo conhecimento sobre doenças sexualmente transmissíveis (DSTs), eles ainda detêm conceitos equivocados sobre sexualidade. Nessa pesquisa, $29,7 \%$ dos alunos e 10,9\% das alunas consideraram homossexualidade uma doença ${ }^{3}$. Ferreira Junior et al. $^{4}$ descreveram certa insegurança entre os internos do quinto ano (I5) e residentes no primeiro ano do programa de clínica médica (R1) da Faculdade de Medicina da USP para tratar desse tema. Em um estudo de auto-avaliação desses indivíduos, notaram-se melhores notas dadas à adoção pessoal de práticas de sexo seguro $(8,7$ e 8,4 respectivamente para I5 e R1) do que a capacidade de orientar pacientes para esse comportamento $(6,7$ e 7,6 respectivamente para I5 e R1). Essa relação foi inversa, por exemplo, ao encontrado para atividade física.

A intersetorialidade é, segundo a Organização Mundial da Saúde (OMS), um dos pontos em torno dos quais a Promoção da Saúde deve se centrar, na medida em que assegurar a saúde exige ação conjunta dos vários setores da sociedade 5 . Essa parceria é suficientemente descrita na literatura médica, com exemplos em ações voltadas para a saúde do trabalhador ${ }^{6}$ e para o ambiente escolar ${ }^{7}$. A importância de ações de promoção da saúde para adolescentes com a temática sexual se evidencia ao verificar-se que o nível de conhecimento sobre o assunto nessa faixa etária por vezes não é adequado ${ }^{8,9}$ e sua vida sexual inicia com maior exposição a riscos ${ }^{10}$.

Além disso, apesar do evidente papel familiar, por vezes esse assunto não é tratado pelos pais com a profundidade necessária, devido a tabus presentes em nossa sociedade ${ }^{11}$. Por outro lado, Camargo e Ferrari ${ }^{8}$ demonstraram que uma ação comunitária realizada em conjunto com uma escola estadual de Londrina, PR, gerou aumento no conhecimento sobre a anatomia feminina e gestação entre estudantes da oitava série do ensino fundamental.

O objetivo do presente artigo é relatar um caso de aplicação de uma ação comunitária, integrando os setores saúde e educação, elaborado com a meta de discutir sexualidade com adolescentes, e executado por alunos do primeiro ano da Faculdade de Medicina da Universidade de São Paulo (FMUSP), professores da disciplina de graduação MSP-0670 "Atenção Primária à Saúde I", agentes comunitários de saúde (ACSs) da Unidade Básica de Saúde Jardim Boa Vista e profissionais da ETEC Raposo Tavares em 2012.

\section{RELATO DO CASO}

\section{A disciplina MSP-0670 “Atenção Primária à Saúde I"}

A disciplina MSP-0670 “Atenção Primária à Saúde I" faz parte do currículo obrigatório do primeiro ano da graduação em medicina da FMUSP, com carga horária de 135h. Esta disciplina tem como objetivos apresentar aos alunos o modelo de atenção primária predominante no país, a Estratégia Saúde da Família (ESF), os conceitos norteadores do SUS e a estrutura e funcionamento de uma UBS. Também procura, a partir de exemplos teóricos e práticos, capacitar os alunos a compreender as várias dimensões da saúde e identificar seus problemas. Envolve também o conceito de comunidade, utilizando-se dela para promover saúde, por meio, por exemplo, de seus equipamentos sociais, como escolas, igrejas, etc.

Os alunos são divididos em cinco grupos, cada um tendo aulas e atuando em uma UBS que faz parte do Projeto Região Oeste ${ }^{12}$ da Fundação Faculdade de Medicina. Esta intervenção foi realizada pelo grupo da UBS Jardim Boa Vista de 2012, durante o segundo semestre do curso.

\section{A ação comunitária "Sexo sem tabu"}

\section{Escolha e delineamento da proposta}

As demandas apresentadas pela UBS Jardim Boa Vista para a intervenção de 2012 foram: a criação de uma brinquedoteca, o ensino de primeiros socorros a professores de uma creche ou um trabalho em conjunto com a ETEC Raposo Tavares, de forma a estreitar o vínculo entre as instituições. Considerando a efetividade $\mathrm{e}$ o legado da ação, foi escolhido o estreitamento de vínculos com a ETEC, abordando o tema sexualidade com seus alunos e discutindo temas como métodos contraceptivos, DSTs, aborto e gravidez.

\section{Preparação}

O grupo de trabalho foi constituído por nove alunos do primeiro ano, seis agentes comunitários de saúde e um professor da disciplina. O objetivo geral foi oferecer ferramentas para que os jovens do primeiro e segundo anos do ensino médio tradicional da ETEC Raposo Tavares tivessem condições de compreender melhor sua própria sexualidade, permitindo uma tomada de decisão autônoma e consciente. Para tanto, foi idealizada uma intervenção que levasse informações com linguagem acessível, em uma discussão aberta, rompendo a ideia de uma transmissão de conhecimentos unidirecional.

O grupo reunia-se semanalmente para a elaboração da intervenção, dentro dos horários disponibilizados pela 
Barros BF et al. Sexo sem tabu.

disciplina e pela gerência da UBS. A presença dos ACSs, conhecedores da demanda daquela área, proporcionava participação ativa nas temáticas abordadas durante a ação comunitária. Além disso, possibilitava um vínculo de longo prazo com os alunos da ETEC.

Iniciamos o trabalho com uma revisão da literatura sobre os tópicos principais ${ }^{1,13-16}$. Optou-se por trabalhar com situações-problema, baseadas em dinâmicas propostas pelo Ministério da Saúde ${ }^{17}$. Foram selecionados alguns textos propostos pelo MS, e acrescentados outros produzidos pelo grupo (Quadro 1). A utilização dessas dinâmicas objetivou incitar a criticidade dos jovens, colocando-os em diversos cenários como protagonistas. Desta forma, eles eram estimulados a responder o que eles fariam na situação apresentada. Além disso, algumas imagens ilustrativas de doenças sexualmente transmissíveis foram escolhidas pelo grupo para serem usadas durante a discussão.

QUADRO 1. Textos utilizados para a dinâmica com os alunos da ETEC Raposo Tavares. Adaptado de texto do Ministério da Saúde ${ }^{17}$ e criação livre do grupo

1. Tenho 15 anos e uma família tradicional. Comecei a namorar um usuário de maconha e, para me sentir incluída do grupo de amigos dele, passei a fazer uso de bebidas alcoólicas sempre que saímos e tenho curiosidades sobre o uso da droga. Por diversas vezes me senti tentada a usar para sentir também o que ele sente. Minha família não aprova nosso namoro, mas eu gosto muito dele e gostaria de ajudá-lo a sair do mundo das drogas.

2. Minha família é muito conservadora e eu comecei a ter relações sexuais com 14 anos com meu namorado, a gente fez algumas vezes sem camisinha, minha mãe desconfiou que eu estivesse fazendo sexo e ficou muito estranha comigo. Acabei tendo um corrimento vaginal, agora não sei se falo com ele, tenho vergonha e medo, não sei se a culpa foi minha ou se peguei dele.

3. Quando conheci meu vizinho, éramos só amigos. Com o passar do tempo acabamos saindo juntos e, hoje, apesar de já ter se mudado, ele vem todos os dias na minha casa. Como está estudando, não quer se prender a ninguém. Só quer transar, mas não somos namorados. Será que se eu transar, ele fica comigo?

4. Alex, 16 anos, namora Marina de 17 anos há quase um ano. Ele está terminando o $2^{\circ}$ grau e está em dúvida se vai para a Universidade ou se começa a trabalhar. Seus pais não são ricos e às vezes até enfrentam dificuldades. Há uma semana, Marina lhe contou que acha que está grávida. Agora Alex tem que tomar uma decisão em sua vida.

5. Cacá, seu amigo de 15 anos, recentemente declarou-se homossexual na escola, surpreendendo a todos. Desde então, passou a sofrer preconceitos e a ser "zoado", sentindo-se rejeitado e triste. Ela está pensando até em abandonar a escola.

6. Meu namorado não quer usar camisinha, mas eu tenho medo de pegar alguma DST ou engravidar. Algumas amigas minhas não usam e nunca pegaram nada. Temo que ele termine comigo se eu obrigá-lo a usar camisinha. E agora?

Para estimular a participação e discussão de dúvidas com todos os adolescentes, inclusive os mais reservados, o grupo propôs a distribuição de pedaços de papel a cada aluno, nos quais cada um teria a possibilidade de escrever, de maneira anônima, perguntas que se relacionassem ao assunto sexualidade. Essas perguntas foram depositadas em uma caixa disponibilizada pelo grupo. Todos foram incentivados a colocar a folha de papel ainda que em branco, desta forma o autor de uma pergunta não seria identificado pelos colegas. Outros temas foram tratados conforme demanda dos alunos. Devido ao uso maciço das redes sociais por adolescentes, o grupo também criou um perfil no Facebook, denominado "Sexo sem Tabu", com o intuito de propiciar sigilo e acessibilidade aos alunos da ETEC, caso houvesse dúvidas que não quisessem expôr aos colegas, ou se as surgissem posteriormente aos dias de intervenção.

Comoobjetivo deavaliaroimpacto daintervenção e ampliar o conhecimento sobre o grupo de adolescentes com os quais se trabalhou, foi criado um questionário com respostas anônimas. Além da sexualidade e impressões sobre a intervenção, informações sobre uso de drogas, realização de exercícios físicos, bullying e outros tipos de violência também foram colhidas.

\section{Execução e avaliação}

A direção da escola reservou dois dias para a execução da Intervenção, um para o primeiro ano do ensino médio e o outro para o segundo ano. A escola aderiu fortemente, dedicando boa parte do período de aula à aplicação do projeto, condição fundamental para a dinâmica proposta. Os alunos da ETEC foram divididos em grupos mistos (meninos e meninas), com cerca de 20 adolescentes em cada um. Dois a três alunos de medicina e um a dois ACSs da UBS Jardim Boa Vista acompanharam cada grupo. Não houve a presença do diretor ou de professores da escola nas salas em que foi realizada a intervenção para manter o sigilo e evitar que os alunos da ETEC ficassem inibidos. Durante o período da intervenção o docente da FMUSP supervisionava periodicamente as discussões para esclarecimento de dúvidas não resolvidas pelo grupo. 
Foi explicada inicialmente aos alunos a proposta da intervenção e distribuídos os pedaços de papel para anotar as dúvidas. Na sequência, houve a aplicação das dinâmicas e discussão das situações-problema. As dúvidas foram recolhidas e respondidas e, ao final, aplicado o questionário. O perfil no Facebook ficou ativo por cerca de um mês.

\section{RESULTADOS}

Dentre os alunos do primeiro e segundo colegial da escola, 118 participaram da intervenção e responderam o questionário anônimo ao final. A mediana de idade foi 16 anos. A Tabela 1 mostra a resposta dos adolescentes a questões relacionadas à intervenção. Adicionalmente, $36(31,6 \%)$ de 114 alunos declararam ter parceiro sexual. Destes, $27(75,0 \%)$ relataram o uso apenas de camisinha como método contraceptivo, $6(16,7 \%)$ camisinha e pílula anticonpcional combinados, $1(2,7 \%)$ apenas pílula anticoncepcional, 1 (2,7\%) injeção trimestral e $1(2,7 \%)$ não utilizava nenhum método. A Tabela 2 traz respostas a outros temas ligados à saúde dos adolescentes.

TABELA 1. Resposta dos adolescentes às questões relacionadas à intervenção

\begin{tabular}{l|c}
\hline Pergunta & Resposta: Sim \\
\hline A discussão sobre sexualidade ajudou a esclarecer algumas dúvidas? & $115 / 117(98,3 \%)$ \\
\hline Você acha que as informações levadas têm relevância para sua vida? & $114 / 117(97,4 \%)$ \\
\hline A discussão trouxe informações que você desconhecia? & $99 / 117(84,6 \%)$ \\
\hline
\end{tabular}

TABELA 2. Resposta dos adolescentes a outras questões ligadas à sua saúde

\begin{tabular}{l|c}
\hline Pergunta & Resposta: Sim \\
\hline Você já fez uso de bebidas alcoólicas? & $82 / 118(69,5 \%)$ \\
\hline Você já fumou cigarros? & $26 / 116(22,4 \%)$ \\
\hline Você já fez uso de outras drogas? & $13 / 118(11 \%)$ \\
\hline Você pratica exercícios físicos regularmente? & $63 / 116(54,3 \%)$ \\
\hline Você já praticou bullying? & $72 / 115(62,6 \%)$ \\
\hline Você já sofreu bullying? & $78 / 116(67,2 \%)$ \\
\hline Você acredita que o bullying seja um ato de violência? & $99 / 114(86,8 \%)$ \\
\hline Você acredita que a violência (verbal ou física) pode deixar marcas na personalidade do indivíduo? & $114 / 117(97,4 \%)$ \\
\hline
\end{tabular}

\section{DISCUSSÃO}

Houve grande adesão dos alunos à proposta de discussão. Adicionalmente, a avaliação da ação revelou que os alunos da ETEC tiveram uma leitura bastante positiva da atividade. Os benefícios não se restringiram aos alunos da ETEC, estendendo-se à aquisição de habilidades e informações pelos universitários.

Uma das principais habilidades em treinamento foi comunicação. A discussão realizada com os estudantes da ETEC exigiu falas claras e atitudes que se adequassem ao público em questão, ainda que o assunto seja, por vezes, delicado. Era necessário abrir espaço para a fala do outro e saber o momento certo de intervir no diálogo, atributo fundamental para o médico.

Falar sobre sexualidade não se restringe a informar sobre doenças sexualmente transmissíveis. Inclui aspectos comportamentais, psicológicos, emocionais e fisiológicos.
Associar prontamente sexualidade apenas a doenças é muitas vezes um viés da graduação, que tem reflexo na conduta adotada por profissionais da saúde ${ }^{2}$, equívoco que o grupo conscientemente procurou evitar. A ação com esses moldes forçou o grupo de trabalho a lidar com suas limitações e contornar dificuldades, inclusive ligadas a situações não previstas durante o delineamento do projeto. A ocorrência dessas situações é motivada pela curiosidade dos indivíduos nessa faixa etária sobre o tema, como ressaltam Carvalho et $\mathrm{al}^{18}$. Os textos utilizados na dinâmica estimularam os jovens a refletirem sobre as situações propostas e enriqueceram a discussão. Alguns associaram os textos a experiências próprias ou de pessoas de seu convívio, trazendo à conversa exemplos reais. Questões relacionadas a sigilo médico e uso de preservativos foram trazidas com frequência pelos alunos do ensino médio. Mostrou-se necessário, desta forma, se preparar para um cenário que não é "pré-fabricado". No grupo, isto foi 
Barros BF et al. Sexo sem tabu.

feito estudando a literatura especializada sobre o tema e discutindo a importância de reconhecer limitações.

A experiência da execução mostrou fortalezas da estratégia. A dinâmica funcionou bem em grupos pequenos (até 15 alunos). O uso das caixas para perguntas anônimas também se mostrou proveitoso. Para que os efeitos da ação não se encerrassem no dia da sua execução, houve participação dos ACSs da UBS Jardim Boa Vista, em cuja área de cobertura se localiza a ETEC. Os jovens também foram orientados a procurar a UBS mais próxima de suas casas. Entretanto, a tentativa de manter um grupo em rede social entre os alunos da ETEC e da Faculdade de Medicina teve pouca adesão, por motivos desconhecidos para o grupo.

Algumas propostas iniciais do grupo não puderam ser postas em prática, devido ao tempo restrito. Havia a intenção de colher informações sobre que assuntos os alunos do ensino médio estariam interessados para expandir a discussão. Além disso, também não conseguimos estender a ação para o terceiro ano do ensino médio ou às turmas do ensino técnico.

Concluindo, esta ação comunitária, com foco em promoção da saúde dos adolescentes e abordando o tema da sexualidade, trouxe resultados positivos para os alunos do ensino médio e para a formação profissional dos estudantes de medicina.

Agradecimentos: Os autores agradecem aos demais alunos da Faculdade de Medicina que realizaram a intervenção, Ac. Bruno de Oliveira Stephan, Ac. Carol Flaksbaum Moll, Ac. Douglas Gouvea e Ac. Esther Pacheco; à coordenadora da disciplina “Atenção Primária à Saúde”, Profa. Dra. Ana Claudia Germani; à UBS Jardim Boa Vista nas pessoas da Dra. Renata Alves de Souza (médica), Enfa. Ana Emília Leal (gerente) e seus agentes comunitários de saúde, ACS Evelyn Frade Mourino, ACS Leonardo Oliveira de Souza, ACS Marcia Cristina Bonfante, ACS Renato Francisco da Conceição, ACS Rosangela dos Santos e ACS Rosangela Rosa da Costa; à equipe da ETEC Raposo Tavares, no nome do seu diretor José Wanderlei Lua da Silva e a todos os alunos da ETEC que participaram das discussões.

\section{REFERÊNCIAS}

1. Brasil. Ministério da Saúde. Secretaria de Atenção à Saúde. Departamento de Atenção Básica. Saúde sexual e saúde reprodutiva. Brasília; 2010. (Série A. Normas e manuais técnicos. Cadernos de Atenção Básica, n. 26) [citado 19 mar. 2013]. Disponível em: http://189.28.128.100/dab/ docs/publicacoes/cadernos_ab/abcad26.pdf.

2. Almeida M. Diretrizes curriculares nacionais para os cursos universitários da área da saúde. Londrina: Rede Unida; 2003.

3. Lima MCP, Cerqueira ATAR. Crenças sobre sexualidade entre estudantes de medicina: uma comparação entre gêneros. Rev Bras Educ Med. 2008;32(1):49-55. http:// dx.doi.org/10.1590/S0100-55022008000100007

4. Ferreira Junior M, Germani ACCG, Oliveira AAP. Selfperception of medical students and residents regarding personal habits, skills, and interest on health promotion and disease prevention. Rev Soc Bras Clin Med. 2011;1:1-10.

5. Lins MJCA. Intersetorialização: atenção básica e promoção da saúde no âmbito do SUS. Diretoria de Promoção da Saúde, Superintendência de Vigilância em Saúde, Secretaria de Estado da Saúde de Alagoas. Maceió;. 2012. Disponível em: http:/www.saude.al.gov.br/sites/default/ files/intersetorializacao___atencao_basica_e_promocao_ da_saude_7_pag_3_de_referencias.pdf

6. Machado JMH, Porto MFS. Promoção da saúde e intersetorialidade: a experiência da vigilância em saúde do trabalhador na construção de redes. Epidemiol Serviços Saúde. 2003;12(3):121-30. Disponível em: http:// www.ins.gob.pe/repositorioaps/0/0/jer/maestria_2012/ Art $\%$ C3\%ADculo\%205.pdf

7. Aerts D, Alves GG, La Salvia MW, Abegg C. Promoção da saúde: a convergência entre as propostas da vigilância da saúde e da escola cidadã. Cad Saúde Pública. 2004;20(4):1020-8. http://dx.doi.org/10.1590/S0102$311 X 2004000400017$.

8. Camargo EAI, Ferrari RAP. Adolescentes: conhecimentos sobre sexualidade antes e após a participação em oficinas de prevenção. Ciênc Saúde Coletiva. 2009;14(3):937-46. http://dx.doi.org/10.1590/S1413-81232009000300030.

9. Duarte CF, Luana BH, Medeiros ML. Avaliação de conhecimento contraceptivo entre adolescentes grávidas em uma Unidade Básica de Saúde do Distrito Federal. J Health Sci Inst. 2012;30(2):140-3. http://www.unip.br/ comunicacao/publicacoes/ics/edicoes/2012/02_abr-jun/ V30_n2_2012_p140-143.pdf

10. Cedaro JJ, Vilas Boas LMS, Martins RM. Adolescência e sexualidade: um estudo exploratório em uma escola de Porto Velho - RO. Psicol Cienc Prof. 2012;32(2):320-39. http://dx.doi.org/10.1590/S1414-98932012000200005

11. Soares SM, Amaral MA, Silva LB, Silva PAB. Oficinas sobre sexualidade na adolescência: revelando vozes, desvelando olhares de estudantes do ensino médio. Esc Anna Nery. 2008;12(3):485-91. http://dx.doi.org/10.1590/ S1414-81452008000300014

12. Faculdade de Medicina da USP. Projeto Região Oeste: ensino, pesquisa, assistência [citado 16 fev. 2013]. Disponível em: http://pro.fm.usp.br.

13. Veronesi R, Foccaccia R. Tratado de infectologia. São Paulo: Atheneu; 2009.

14. Rios INC, Mercês Junior SQ, Rios Filho INC. DST na prática diária. Salvador: Ed. Helvétia; 2002. 
15. Hinrichsen SL. Doenças infecciosas e parasitárias. Recife: Ed. Guanabara Koogan; 2005.

16. Brasil. Ministério da Saúde. Departamento de Atenção Básica. HIV/Aids, hepatites e outras DST. Brasília; 2006. (Cadernos de Atenção Básica, n. 18) [citado 19 mar. 2013]. Disponível em: http://189.28.128.100/dab/docs/ publicacoes/cadernos_ab/abcad18.pdf.

17. Brasil. Ministério da Saúde. Secretaria de Políticas de
Saúde. Coordenação Nacional de DST e Aids. Manual do multiplicador: adolescente. Brasília; 2000 [citado 15 out. 2012]. Disponível em: http://pt.scribd.com/doc/18055084/ dinamicas-para-jovens-Sexualidade.

18. Carvalho AM, Rodrigues CS, Medrado KS. Oficinas em sexualidade humana com adolescentes. Estud Psicol. 2005;10(3):377-84. 294X2005000300006
http://dx.doi.org/10.1590/S1413- 